Unfallchirurg $2010 \cdot 113: 770$

DOI 10.1007/s00113-010-1797-5

Online publiziert: 18. Juli 2010

(c) Springer-Verlag 2010
H. Siebert

Generalsekretär, Deutsche Gesellschaft für Orthopädie und Unfallchirurgie e.V;;

Deutsche Gesellschaft für Unfallchirurgie e.V., Berlin

\title{
Versorgungsstruktur in Deutschland
}

\section{Kommentar zum Beitrag}

Krettek C (2010) Traumasysteme in Deutschland, USA und Australien - ein internationaler Vergleich. Unfallchirurg 113:771-777, DOI: s00113-010-1796-6

Liebe Kolleginnen und Kollegen,

zunehmend werden Vorschläge zur Änderung unserer Versorgungsstrukturen (z. B. Schaffung eines Facharztes für Notfallmedizin, Notfallchirurgie und andere) mit dem Hinweis auf die oft jahrzehntelange und erfolgreiche Umsetzung in anderen, v. a. angloamerikanischen Ländern diskutiert und zur baldigen Umsetzung - möglichst $1 \mathrm{zu} 1$ - empfohlen. Dabei wird häufig übersehen, dass diese Länder nicht nur eine völlig andere Struktur, Finanzierung, „Kultur“ und Zielsetzung ihres Gesundheitswesens haben, sondern sich auch erheblich in den Versorgungsstrukturen und -formen z. B. ambulante/stationäre Facharztvorhaltung - von unserem System unterscheiden.

\section{(7) Lassen sich angloamerikanische Versorgungstrukturen auf Deutschland übertragen?}

Damit können die dort u. U. sehr erfolgreichen Versorgungsformen bzgl. Optimierung der Patientenbetreuung und versorgung nicht auf unsere Verhältnisse übertragen und Studienergebnisse mit entsprechender Evidenz nicht als in
Deutschland ebenso evident und damit beweiskräftig angesehen werden.

Mit dem Beitrag von C. Krettek wollen wir Ihnen die Strukturen und Praxis der Unfallversorgung in Australien und den USA - auch aus eigenem intensivem Erleben - vorstellen und damit auch die Unterschiede in den Gesundheitssystemen beleuchten.

Ihr

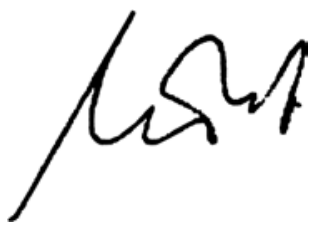

H. Siebert

\section{Korrespondenzadresse}

Prof. Dr. H. Siebert

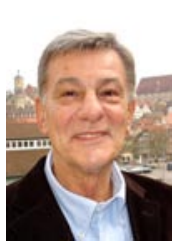

Generalsekretär,

Deutsche Gesellschaft für

Orthopädie und

Unfallchirurgie e.V.;

Deutsche Gesellschaft für

Unfallchirurgie e.V.

Luisenstr. 58, 10778 Berlin

hsiebert@diaksha.de 